\author{
2022, Volume 9, ID 602 \\ DOI: 10.15342/ijms.2022.602
}

\title{
PERSPECTIVES
}

\section{Music, Arts and Cognitive Behavioural Therapy to Treat Sleep Disorders}

\author{
Wolfgang Mastnak \\ Beijing Normal University, China ; University of Music and Performing Arts Munich, Germany
}

\section{ABSTRACT}

Insomnia is a global burden. Cognitive-behavioural therapy models for treating pathological sleeplessness have significant effect sizes, hence the common recommendation of CBT-I. Akin to this approach, the present article presents a novel model which relates to the broader spectrum of sleep disorders and involves music- and arts-specific health benefits such as (i) vocal rituals as soothing sleep cues and means to make irritating cognitions fade, (ii) control of sleepiness and inappropriate daytime naps through music-based martial arts techniques, (iii) voice- and movement-based relaxation and psychosomatic re-balancing, as well as profound music immersion and music-induced altered states of consciousness, (iv) arts-based psychotherapy to cope with pathological factors, such as nightmare objects and (v) psychoeducation to understand the benefits of the arts for healthy sleep. Cultural features of sleep habits, differences between face-to-face and online practices, individualized arts-based CBT, and the multidimensional healing power of the arts are discussed and encourage further research to optimize personalized and standardized arts-based cognitive behavioural therapy in sleep medicine and public health.

\section{Interdisciplinary research}

- Evidence-based epistemology: quantitative, qualitative, individualised, trans-methodological

- Underlying mechanisms: neurophysiological, psychological, complementary \& integrative

- Sociocultural perspectives: anthropological, sociological, ethnological

- Arts \& aesthetics: epistemology of arts and arts-related philosophy of health

\section{$\mathbf{1}$}

Arts-based cognitive behavioural therapy of sleep disorders ACBT-S: framework, individualisation, settings

Relevant arts e.g. Northeastern Cradle Songs 东北摇篮曲

- Stimulus control \& sleep cues, e.g. musical bedtime rituals or soothing calligraphy

- Sleep restriction, e.g. creative martial arts or vocal activation

- Psychosomatic relaxation, e.g. Sound-balancing or Guqin autohypnotherapy

- Psychotherapy, e.g. music-induced imagery techniques

- Psychoeducation, e.g. about music, sleep and the Orexin-system

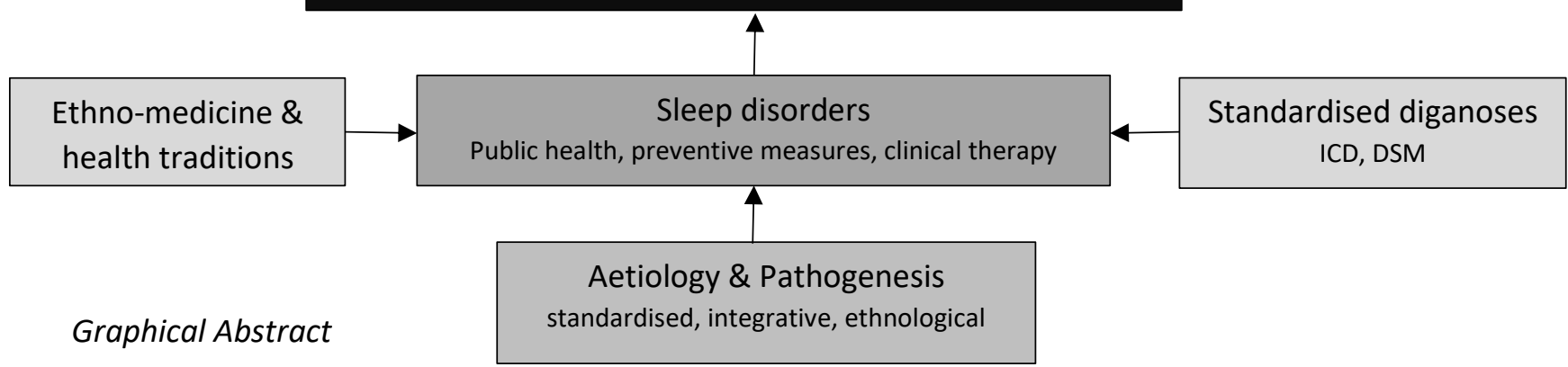

KEYWORDS: Music, Cognitive Behavioural Therapy, Insomnia.

Correspondence: Prof. Wolfgang Mastnak, Address: Beijing Normal University; University of Music and Performing Arts Munich. Copyright () 2022 Mastnak W. This is an open access article distributed under the Creative Commons Attribution 4.0 International, which permits unrestricted use, distribution, and reproduction in any medium, provided the original work is properly cited. 


\section{INTRODUCTION}

Healthy sleep is commonly regarded as a key factor of health, although notions such as 'sleep health' [1] refer to different definitions and opinions, hence the call for clarification. Integrative sleep medicine is faced with the inner connection between sleep hygiene, mental health, and wellness in work and occupational therapy [2].

Promoting culturally sensitive music therapy, clinical research at the Beijing Normal University Research Centre of Arts Therapies focuses mainly on Guqin music therapy to alleviate affective and psychosomatic conditions in gastric cancer patients, including sleep disturbances. Due to the high prevalence of sleep disorders, researchers call sleep epidemiology a rapidly growing field [3], where sleep disorders are not only bi-directionally connected with psychiatric disorders such as depression [4]. In other words, both risk factors and symptoms [5] and frequent sequelae and complications of other illnesses such as cancer [6].

Sleep disorders form a multifaceted field of integrative pathology, hence the necessity to provide precisely shaped treatment plans for comorbid sleep disturbances in migraine [7]. Appropriate treatment is a crucial issue that involves pharmacological, psychological, and sociocultural considerations. Medication such as benzodiazepines and Z-drugs - i.e., non-benzodiazepine agonists, e.g., zolpidem and zopiclone - may be used for short-term treatment of insomnia. However, it entails risks of tolerance and dependence, while meta-analyses have shown high effect sizes of side-effect-free cognitive behavioural therapy [8].

Due to significant clinical benefits, cognitive behavioural therapy has not only become an important approach in the realm of sleep medicine: the American College of Physicians (ACP) even calls it the first-line treatment for insomnia, and 'CBT-I' has become a label [9]. In general, practice guidelines recommend cognitive behavioural therapy for insomnia (CBT-I) and point out that hypnotic medication shall be considered if CBT-I does not work. Moreover, compared with pharmacotherapy, CBT-I has been shown to be superior in reducing insomnia symptoms and maintaining sleep improvements for years. Furthermore, patients often prefer non-pharmacological approaches as they are considered to be better at improving daytime functioning and less likely to produce adverse side effects [10].

CBT-I is typically based on four cornerstones: (i) stimulus control, such as pairing the bed with sleep and leaving the bed when awake. Patients are encouraged to change habits that make sleeping difficult in this context. Such as eating, watching TV or using smartphones, and using the bedroom - at least during treatment - exclusively as a place for restful sleep and sex [11]; (ii) sleep restriction and compression, which limits time spent in bed to re-establish a consistent sleep schedule and to increase the drive to sleep as well as temporary daytime fatigue. Although CBT-I may also be recommended for psychiatric patients with insomnia [12], we have to consider possible pathogenic sleep reduction interactions with core symptoms of psychiatric disorders. By way of illustration, sleep restriction may have adverse impacts on patients with bipolar disorder or seizures; (iii) cognitive therapy and psychoeducation to change irritating cognitive patterns. E.g., alerting thoughts about sleep loss and helping patients understand mechanisms of insomnia. Alongside the importance of good sleep hygiene; (iv) relaxation training, which may comprise breathing exercises, progressive muscle relaxation, autogenic training, biofeedback methods, and even certain forms of meditation or hypnotherapy.

Clinical experiences and observations suggest that musicand arts-related interventions may facilitate such therapeutic processes, hence the present therapeutic model. Recently, the Beijing Moresleep Healthcare Technology Company - in China, also known as 慕眠, literally 'to adore sleep" - has begun to provide an individualized music-based program for people who have insomnia. This music-based cognitive and behavioural therapy for insomnia, shortly MCBTi, is a 6-weekprogram called 'Sleepower' consisting of six modules: 'sense of sleep security establishment, sleep restriction, stimulus control, relaxation training, sleep hygiene improvement and neutral system rebalance' [cited from informal information of the company to the author].

Although there are similarities between 'Sleepower' and the present model, there are also significant differences:

1. The present model is music-based and encompasses other artistic media such as calligraphy or martial arts.

2. According to international manuals such as ICD and DSM, it is not limited to insomnia but relates to the broad spectrum of sleep disorders, e.g., parasomnias.

3. Cultural sensitivity takes the patient's acculturation into account and adapts efficient elements of traditional techniques.

Moreover, the construction of the present model used comparative research as well as techniques of systemic meta-synthesis [13].

\section{THE COGNITIVE-BEHAVIOURAL MUSIC-ARTS} MODEL

The present model has to be understood as an open system rather than an invariant set of techniques. Clinical experience suggests that integration of cognitivebehavioral and cultural approaches may improve therapeutic outcomes, particularly in patients with a strong attachment to aesthetic and creative practices.

\section{Stimulus control}

While CBT-I usually focuses on the avoidance of irritating stimuli such as smartphones in bed, the present model suggests also consider sleep-inducing stimuli such as bedtime rituals with soothing music, sound-meditation, or the hypnotic power of traditional calligraphy with dreamlike contents.

\section{Sleep restriction}

While CBT-I recommends daytime wakefulness, transitions between sleep attacks in the sense of narcolepsy type 2 [14], excessive sleepiness, and inappropriate habits of napping are known and form multi-faceted syndromes. Although the present music-arts-oriented model agrees with the avoidance of sleep, which is likely to disturb the natural circadian rhythm, it also recommends distinct practices to cope with sudden attacks of sleepiness such as the individualized use of Chinese martial arts, e.g. 'musicbased eight pieces of brocade' (音乐八段锦), vocal adaptations of the Kung Fu model 'Five animal play' (五 禽戏) or combined breathing and vocal techniques for mental activation and 'ignition.' 


\section{Psychosomatic relaxation}

Although CBT-I provides a broad spectrum of efficient relaxation techniques, some patients may especially profit from arts-based techniques such as sound-based Zenmeditation, focused listening [15], which is a well-defined mode of auditory immersion, Guqin-guided auto-hypnosis (the Guqin 古琴 is the classical Chinese zither with a history of 5000 years) or elements of the voice-body-based models Sound Balancing and Sound Energising [16]. Sleep-obstructing obsessive-compulsive cognition may fade, and patients experience the encouraging feeling of self-efficacy: while sleep disturbance has been harming their health and self-image, artistic techniques help them control their sleep disorder and regain good sleep. Moreover, patients also say that these aesthetic and creative activities have improved their quality of life and not only relieved or removed symptoms.

\section{Psychoeducation and psychotherapy}

CBT-I recommends Psychoeducation to help patients understand symptoms and underlying mechanisms of insomnia. Meanwhile, Psychoeducation has also gained ground in psychiatric and psychosomatic music therapy and clinical music education [17]. Being also recommended in the present model, Psychoeducation may help patients to understand specific impacts of music activities and aesthetic experiences on sleep processes and sleep quality, such as the dynamic connections between the music-generating central-nervous system and neurophysiological sleep-wake-mechanisms of the hypocretin/orexin system [18].

As the present therapeutic model relates to the whole spectrum of sleep disorders, arts-based psychotherapeutic approaches gain importance. These may include musicguided imagination techniques to cope with fears and the contents of nightmares, enhanced creativity to develop individualized conditions for healthy sleep, or musicinduced trance to discover unconscious irritations of sleep.

\section{DISCUSSION}

Experiences with arts-based practices to alleviate sleep disturbances and comparative research on CBT-I raise several questions and call for further studies to optimize non-pharmacological sleep therapy.

Given that the actual core of sleep-improving interventions is performed at home and without immediate professional support, our integrative sleep therapeutic program also involves complex learning processes, particularly competence of self-application and self-regulation, hence the necessity to also take individualized psychology of learning into account.
Both CBT-I and the present cognitive-behavioral musicarts model for sleep disorders refer to a broad spectrum of factors and therapeutic components, such as stimulus control and relaxation. According to clinical analyses, however, individualized therapeutic programs have to assess their 'therapeutic weight' alongside the time factor. In other words, therapeutic programs have to be precisely tailored to the characteristics of individual sleep disturbances, which limits possibilities of absolute standardization.

Particularly geographical and epidemiological conditions have encouraged online therapies as well as comparative research about differences from classical fact-to-face interventions. Advanced forms of arts-based online therapies may not only improve equal rights in public health and medical treatment but also bring about novel modes of treatment structures such as short online interventions before going to bed.

Arts-oriented sleep therapy also has to respect cultural differences, which concern both a patient's acculturation and sociocultural traditions such as the Spanish siesta. In this context, also bedtime rituals such as soothing songs, imaging techniques, or arts-based meditation form an important research object.

Public health distinguishes between the promotion of health, preventive measures, and clinical therapy. Although these areas are basically different, they are systemically interconnected and relevant to the selfapplication of arts-based models to improve sleep quality in different areas such as occupational medicine (prevention of stress-associated sleep disturbances) or music education (mental health and good sleep in pupils). Aetiological analyses and clear identification of pathogenic factors are standard in medicine and also apply to sleep disorders. By way of illustration, stress- or burnout-related fatigue may cause not only severe insomnia but also inhibit the successful application of the interventions mentioned above. Consequently, specific, possibly also arts-based, pre-intervention is required.

Last but not least, we focus on the healing power of aesthetics, a key issue that relates to very different fields such as evidence-based medicine, neuro-aesthetics, or Daoist notions of harmony. To unearth the therapeutic potential of the arts to treat sleep disorders is still an interdisciplinary challenge.

\section{ACKNOWLEDGMENTS}

None.

\section{FUNDING SOURCES}

None. 


\section{REFERENCES}

[1] Buysse DJ. Sleep health: can we define it? Does it matter? Sleep. 2014; 37(1): 9-17. DOI: 10.5665/sleep.3298

[2] [2] Magnavita N, Garbarino S. Sleep, health and wellness at work: A scoping review. Int J Environ Res Public Health. 2017; 14(11): 1347. DOI: 10.3390/ijerph14111347

[3] Ferrie JE, Kumari M, Salo P, Singh-Manoux A, Kivimäki M. Sleep epidemiology - a rapidly growing field. Int J Epidemio. 2011; 40(6): 1431-7. DOI: $10.1093 /$ ije/dyr203

[4] Fang H, Tu S, Sheng J, Shao A. Depression in sleep disturbance: A review on a bidirectional relationship, mechanisms and treatment. J Cell Mol Med. 2019; 23(4): 2324-32. DOI: $10.1111 / \mathrm{jcmm} .14170$

[5] Steiger A, Pawlowski M. Depression and sleep. Int Jo Mol Sci. 2019; 20(3): 607. DOI: 10.3390/ijms20030607

[6] Mogavero MP, DelRosso LM, Fanfulla F, Bruni O, Ferri R. Sleep disorders and cancer: State of the art and future perspectives. Sleep Med Rev. 2021; 56: 101409. DOI: $10.1016 /$ j.smrv.2020.101409

[7] Rains JC. Sleep and migraine: assessment and treatment of comorbid sleep disorders. Headache. 2018; 58(7): 1074-91. DOI: $10.1111 /$ head.13357

[8] [8] Rémi J, Pollmächer T, Spiegelhalder K, Trenkwalder C, Young P. Sleep-related disorders in neurology and psychiatry. Dtsch Arztebl Int. 2019; 116(41): 681-8. DOI: 10.3238/arztebl.2019.0681

[9] Koffel E, Bramoweth AD, Ulmer CS. Increasing access to and utilization of cognitive behavioral therapy for insomnia (CBT-I): a narrative review. J Gen Intern Med. 2018; 33(6): 955-62. DOI: 10.1007/s11606-018-4390-1
[10] Pappa EJ, Quinn JA, Murray JJ, Davidson JR, Ó Brádaigh CM, McCarthy ED. Cognitive behavioural treatment for insomnia in primary care: a systematic review of sleep outcomes. Polymers (Basel). 2021; 13(23): 4103. DOI: 10.3399/bjgp19x705065

[11] Bootzin RR, Epstein DR. Understanding and treating insomnia. Annu Rev Clin Psychol. 2011; 7: 435-58. DOI: 10.1146/annurev.clinpsy.3.022806.091516

[12] Jansson-Fröjmark M, Norell-Clarke A. Cognitive Behavioural Therapy for insomnia in psychiatric disorders. Curr Sleep Med Rep. 2016; 2(4): 233-40. DOI: $10.1007 / \mathrm{s} 40675-016-0055-\mathrm{y}$

[13] Mastnak W. Systemic Meta-Synthesis. ResearchGate. 2021. DOI: 10.13140/RG.2.2.25103.30886

[14] Baumann-Vogel H, Schreckenbauer L, Valko PO, Werth E, Baumann CR. Narcolepsy type 2: A rare, yet existing entity. J Sleep Res. 2021; 30(3): e13203. DOI: $10.1111 /$ jsr. 13203

[15] Mastnak W, Köhler-Massinger D. Modes of music listening to modulate stress and prevent burnout. Musik-, Tanz- und Kunsttherapie. 2017; 27(2): 123-8.

[16] Mastnak W. Sound Work: Voice and body in psychiatry, psychosomatics and health promotion. Musik-, Tanz- und Kunsttherapie. 2018; 28(1), 109-21.

[17] Mastnak W, Tièschky T. Musik-Psychoedukation. Pädagogische und psychiatrische Perspektiven. Diskussion Musikpädagogik. 2017; 75(3): 5-8.

[18] Ebrahim IO, Howard RS, Kopelman MD, Sharief MK, Williams AJ. The hypocretin/orexin system. J R Soc Med. 2002; 95(5): 227-30. DOI: 10.1258/jrsm.95.5.227 